Subscriber access provided by Caltech Library

\title{
Article
}

\section{Local Structure and Bonding of Carbon Nanothreads Probed by High-Resolution Transmission Electron Microscopy}

Stephen J. Juhl, Tao Wang, Brian Vermilyea, Xiang Li, Vincent H. Crespi, John V. Badding, and Nasim Alem J. Am. Chem. Soc., Just Accepted Manuscript • DOI: 10.1021/jacs.8b13405 • Publication Date (Web): 05 Apr 2019

Downloaded from http://pubs.acs.org on April 8, 2019

\section{Just Accepted}

"Just Accepted" manuscripts have been peer-reviewed and accepted for publication. They are posted online prior to technical editing, formatting for publication and author proofing. The American Chemical Society provides "Just Accepted" as a service to the research community to expedite the dissemination of scientific material as soon as possible after acceptance. "Just Accepted" manuscripts appear in full in PDF format accompanied by an HTML abstract. "Just Accepted" manuscripts have been fully peer reviewed, but should not be considered the official version of record. They are citable by the Digital Object Identifier (DOI®). "Just Accepted" is an optional service offered to authors. Therefore, the "Just Accepted" Web site may not include all articles that will be published in the journal. After a manuscript is technically edited and formatted, it will be removed from the "Just Accepted" Web site and published as an ASAP article. Note that technical editing may introduce minor changes to the manuscript text and/or graphics which could affect content, and all legal disclaimers and ethical guidelines that apply to the journal pertain. ACS cannot be held responsible for errors or consequences arising from the use of information contained in these "Just Accepted" manuscripts. 


\section{INTRODUCTION}

Carbon nanothreads, predicted independently in 2001,1 $2011,{ }^{2}$ and 2014, ${ }^{3}$ are $\mathrm{sp}^{3}$-bonded one-dimensional (1D) nanomaterials synthesized by slow polymerization of solid benzene at high pressure. ${ }^{4}$ They assemble into closepacked near-hexagonal crystals ordered across hundreds of microns $^{5}$ (even when the benzene precursor is polycrystalline) apparently guided by a uniaxial component to the applied pressure and packing constraints of rod-like objects under pressure. Many fullysaturated "degree- 6 " 6 and partially-unsaturated "degree$4^{\prime \prime 7}$ nanothread structures have been enumerated by group theoretical analysis. Nanothreads are chemically versatile:8-9 they can be formed from heteroaromatic molecules such as pyridine ${ }^{10}$ and their exterior hydrogens can be substituted without disrupting the carbon backbone, ${ }^{11}$ in contrast to $\mathrm{sp}^{2}$ carbon nanotubes whose sidewall functionalization requires a change in carbon hybridization. Nanothreads are thus both nanomaterials and hydrocarbon molecules..$^{12}$ Their properties have been extensively investigated computationally. For example, they may uniquely combine extreme strength with flexibility and resilience, ${ }^{13-15}$ have higher load transference to polymer matrices than carbon nanotubes due to more irregular surface topography,,$^{16-17}$ and may support torsional deformations $\sim 3$ times those of $\mathrm{sp}^{2}$ nanotubes for which flattening degrades mechanical performance. ${ }^{17}$ Modeling also suggests certain nanothreads may behave as resonators with higher quality factors than carbon nanotubes, ${ }^{18}$ could exhibit electronic properties tunable by tensile strain, ${ }^{19}$ and may show superplastic behavior. ${ }^{20}$ Their thermal conductivity is predicted to be tunable depending on axial structural order.21-22 The ability of narrow, stiff, ${ }^{13}$ straight nanothreads to spatially organize both interior heteroatoms and exterior groups relative to each other may be broadly useful in application areas such as the life sciences, photovoltaics, battery materials, and catalysis. ${ }^{12,23}$ As hydrocarbons, nanothreads can likely be made more robust against degradation (e.g. 
photodegradation and environmental chemical attack) than can other stiff 1D molecules that can organize attached chemical functions (e.g. DNA, cellulose, etc.)., ${ }^{4,24}$ 25 Similar to hydrogen-terminated diamond surfaces, nanothreads are expected to have a negative electron affinity, which may make them attractive for field emission applications. ${ }^{4}$ All of these properties are sensitive to the details of the nanothread structure at length scales from atomic to micron, motivating multidisciplinary structural investigations.

Nanothreads have been studied by $x$-ray diffraction, ${ }^{4-5}$ which reveals near-six-fold diffraction patterns characteristic of pseudohexagonal crystals with an $a$-axis lattice parameter of $6.47 \AA$. The reduced real-space pair distribution function indicates a spatial correlation length of about $15 \mathrm{~nm}$ for translational order in the a-b plane, i.e. perpendicular to the nanothread axes. The fraction of $\mathrm{sp}^{3}-$ bonded carbon is $75 \%$ to $80 \%$ according to nuclear magnetic resonance (NMR) spectroscopy. ${ }^{4,26}$ NMR also reveals that nanothread crystals have fully saturated degree- 6 regions at least $2.5 \mathrm{~nm}$ long. Degree- 6 threads comprise approximately a third of that sample, with partially saturated degree- 4 threads containing $\mathrm{sp}^{2}$ carbon comprising another third. NMR also provides intriguing suggestions of a 4+2-based reaction pathway. ${ }^{26-27}$ Although there is evidence of defect sites such as ortho-, meta-, or para-coordinated phenyl rings, there is no evidence for well-organized non-thread minority phases. The details of the spatial arrangement of degree- 4 and degree- 6 threads relative to each other is not yet known. Preliminary transmission electron microscopy (TEM) imaging of nanothreads shows parallel striations with 6.4$\AA$ spacing, larger than the $5.6-\AA$ spacing expected for the hexagonal $\{10 \overline{10}\}$ planes of nanothreads with a $6.47-\AA \AA a-$ axis lattice constant, with no information about axial order or interthread registry. ${ }^{4}$

Electron microscopy plays a seminal role in characterizing the structure and bonding of new carbon nanomaterials ${ }^{28-29}$ by directly imaging unique modes of structural organization. Here we examine the structure of carbon nanothread lattices at $\mu \mathrm{m}$ to sub-nm length scales using low-dose high-resolution electron microscopy (HREM) imaging and electron diffraction, and also examine their chemical bonding using electron energyloss spectroscopy (EELS). We observe atomic columns attributable to threads that span a substantial fraction of the $80-100 \mathrm{~nm}$ sample thickness and characterize their packing at the level of individual threads. A single point defect disrupts long-range translational order in a 1D system; thus, it is significant that we observe for the first time layer lines in electron diffraction on samples aligned at a non-zero angle to the thread axis: i.e. nanothreads have axial translational order over at least a few nanometers.

\section{EXPERIMENTAL}

Carbon nanothreads were imaged using an FEI Titan ${ }^{3} \mathrm{G} 2$ high-resolution TEM equipped with a spherical aberration-corrected lens and an electron monochromator (see Supporting Information). Nanothread samples were prepared for TEM imaging and spectroscopy by crushing in an agate mortar with cyclohexene or liquid nitrogen (both gave similar results) and drop-casting onto lacey carbon grids. As hydrocarbons, nanothreads are susceptible to electron beam damage, although less so than conventional polymers such as polyethylene $\mathrm{e}^{30-32}$ apparently due to the robustness of their carbon backbone. The 2D pseudohexagonal packing of nanothreads was observed to expand for doses above 30 electrons/ $\AA^{2}$. Thus, we used a low electron dose from a Nelsonian illumination scheme in combination with the monochromator aperture to minimize beam damage..$^{33-35}$

\section{RESULTS AND DISCUSSION}

The carbon K-edge of the EELS spectrum at $284 \mathrm{eV}$ acquired from the edge of a nanothread particle can be fit by three Gaussian peaks associated with the $1 s-$ to $-\pi^{*}\left(\mathrm{sp}^{2}\right)$, $1 \mathrm{~s}$-to- $\sigma^{*}\left(\mathrm{sp}^{3}\right)$, and $1 \mathrm{~s}$-to- $\sigma^{*}\left(\mathrm{sp}^{2}\right)$ transitions (Figure 1$){ }^{36}$ The $\mathrm{sp}^{2}$ and $\mathrm{sp}^{3}$ contents estimated by the two-window method with a glassy carbon $\mathrm{sp}^{2}$ standard $^{37}$ (Figure S1) are $27 \%$ and $73 \%$, respectively, consistent with the range determined by solid-state NMR. ${ }^{4}$ This level of agreement between a highly local probe (TEM) and a global probe (ss-NMR) suggests reasonable sample uniformity. NMR reveals that the $\mathrm{sp}^{2}$ carbon is associated with degree-4 nanothreads and a small fraction of benzene linkers. The thickness of several samples was estimated to be $80-100$ $\mathrm{nm}$ using the log-ratio method in EELS (see Supporting Information).

Nanothreads pack into pseudohexagonal crystals hundreds of microns across.,10 In view of this azimuthal packing order around their common axis, they are not strictly "fibers" as the term is used in diffraction from aligned (bio)polymers, since those generally lack longrange order transverse to the fiber axis. ${ }^{38}$ Identical threads in axial registry with each other would have full threedimensional order. But the constituent threads within a pseudohexagonal packing may suffer variable axial or azimuthal shifts relative to each other, i.e. "disregistry" as 
2 in Figure 2 (and may also be a heterogeneous packing of different degree- 4 and degree- 6 threads). Such disregistry is often found in crystals whose components can readily rotate or translate relative to each other, as for adamantane, $\mathrm{C}_{60}$, and $\mathrm{sp}^{2}$ carbon nanotubes. ${ }^{39}$ Figure 3 shows models of registry and disregistry in orthographic projection for $4 \times 4$ supercells of the stiff-chiral-3 $(136425$ in the nomenclature by Xu et al. ${ }^{6}$ ) nanothread, which is a degree- 6 structure suggested by NMR analysis. ${ }^{26-27}$

We observe a pseudo-hexagonal ${ }^{5}$ array of reflections in selected-area electron diffraction (SAED) patterns collected along the nanothread crystal c-axis (Figure 4a); the rapid decrease of intensity out to fifth order is consistent with a near-cylindrical form factor ${ }^{39}$ while the singular orientation of the reflections indicates that the nanothread pseudohexagonal packing is single-crystalline on the 1-2 $\mu \mathrm{m}$ size scale of the particles examined. The interplanar spacings of the first-order reflections in SAED patterns are $5.7 \pm 0.1 \AA$, which is consistent with the spacings predicted for several candidate degree- 6 nanothread structures (Figures S2-S5), when accounting for thermal expansion and beam-induced dilation of the lattice; these include the structures favored by a detailed solid-state NMR analysis. ${ }^{26-27}$ The other reflections in Figure $4 a$ index close to the

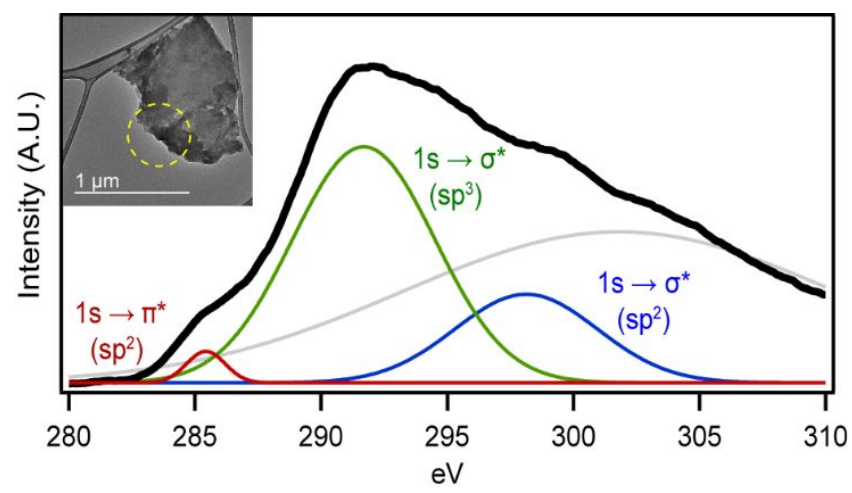

Figure 1. Nanothread Carbon K-edge Electron Energy-Loss Spectrum. Gaussian peaks fit to the spectra can be assigned to the $1 \mathrm{~s}-$ to $-\pi^{*} \mathrm{C}=\mathrm{C}$ (red), $1 \mathrm{~s}-$ to- $\sigma^{*} \mathrm{C}-\mathrm{C}$ (green), and $1 \mathrm{~s}-$ to $-\sigma^{*}$ $\mathrm{C}=\mathrm{C}$ (blue) transitions. The inset is a TEM image of the nanothread particle on which these spectra were collected. A dashed circle shows the area covered by the electron beam during the EELS scan.

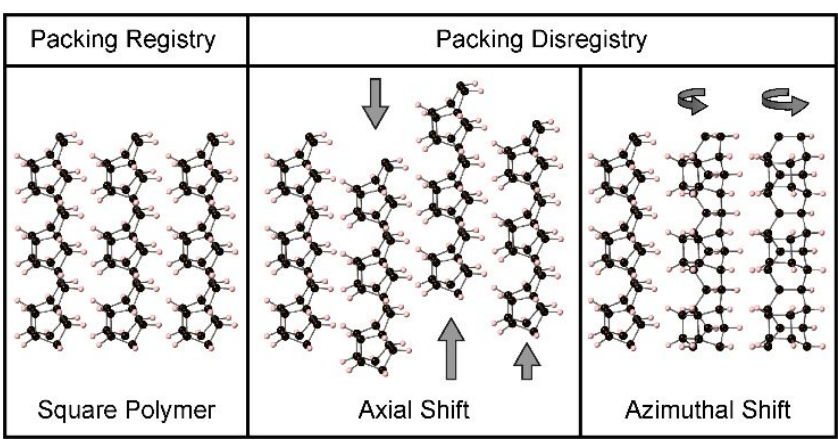

Figure 2. Nanothread Registry and Disregistry. Nanothreads - in this case square polymer $(143562)^{6}-$ in registry form three-dimensionally ordered crystals (left). Arbitrary axial shifts (middle) and azimuthal shifts (right) cause disregistry to give crystals that have ordered hexagonal packing in the $a-b$ plane but are not three-dimensionally ordered.

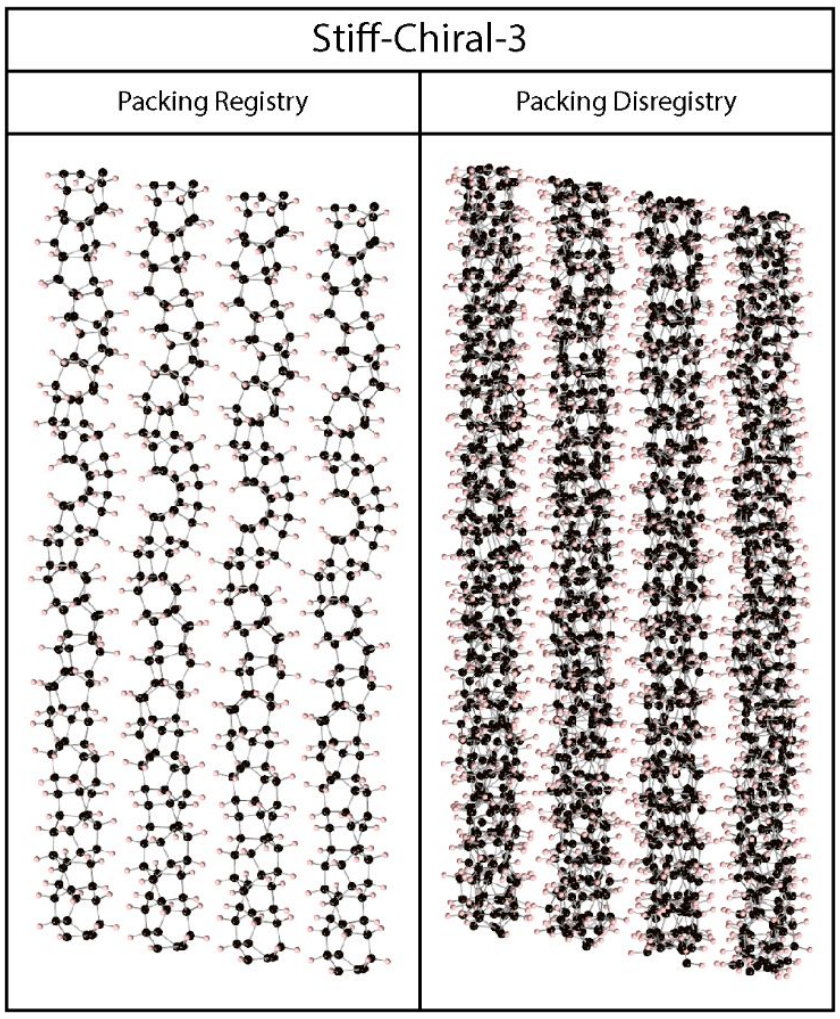

Figure 3. Nanothread Registry and Disregistry. Optimized geometries for $4 \times 4$ supercells of axially ordered stiff-chiral- 3 $(136425)^{6}$ nanothreads with and without interthread packing registry in orthographic projection, for use in TEM simulations. All threads were originally packed hexagonally in a rectangular sixteen-thread supercell either with the same axial offset and azimuthal angle (packing with registry) or with randomly distributed azimuthal angles from 0 to $2 \pi$ and axial shift within c-axis periodicity (packing with disregistry), and then relaxed using the AMBER empirical potential 
implemented in LAMMPS. ${ }^{40-41}$ Note that the threads are tilted slightly with respect to the c-axis after optimization.

$\{1 \overline{1} \overline{0} 0\},\{20 \overline{2} 0\},\{21 \overline{3} 0\}$, and $\{30 \overline{3} 0\}$ families of planes of a hexagonally-packed bundle of nanothreads, while the 57$62^{\circ}$ angles of the $\{20 \overline{2} 0\}$ reflections (Figure S5) are pseudohexagonal, like those observed in $x$-ray diffraction. ${ }^{5}$

Beyond fifth order in the diffraction pattern in Figure 4a, there are no distinct reflections in the experimental SAED patterns. Further pushing the detector to the limits of exposure and reducing the camera length to the edge of the observable range reveals a weak diffuse ring at higher order ( $d \approx 1.2 \AA$, Figure $4 a$ and Figure S5). Diffuse scattering is dominated by a hexagonal envelope that intersects the most intense diffraction spots (i.e. up to fifth order). This diffuse hexagonal envelope is different from the circular Debye-Scherrer rings that would be expected from a secondary amorphous phase. An amorphous phase is not evident in the electron diffraction patterns nor in the NMR spectra. ${ }^{26}$ In contrast, disregistry in nanothread packing should give a diffuse background that follows the overall lattice symmetry, as is observed for adamantane and $\mathrm{C}_{60}{ }^{42}$ Extensive diffraction simulations along [0001] were performed each with a single thread type within a supercell large enough to capture some of the effects of disregistry, shown in Figures S6-S8 plus one illustrative case in Figure $4 \mathrm{~b}$ and Figure $4 \mathrm{c}$. The diffraction simulations reveal two distinct envelope shapes: hexagonal and starlike. Hexagonal shapes arise for chiral threads, especially under disregistry, whereas star-like shapes arise for most of the achiral threads. In particular, several achiral threads with low-order azimuthal rotational symmetry (i.e. $\mathrm{C}_{6}$ symmetry or less) exhibit star-like diffuse diffraction patterns because of "lumpy" cross-sections that produce a discrete set of six azimuthal thread orientations aligned to the underlying pseudo-hexagonal lattice. In contrast, chiral threads are typically more nearly circular in projected cross-section and pack with essentially random azimuthal angles. Especially under disregistry, these threads can produce a hexagonal scattering envelope similar to experiment (see for example stiff-chiral-3 in Figure $4 \mathrm{~b}$ and the achiral 5-8 polymer of Figure S7). However, we suspect that sufficient on-thread structural disorder or mixed thread populations may disrupt the discretization in the azimuthal orientations of the achiral threads to produce similar hexagonal envelopes, although this remains a topic of future investigation.

The finite arc length of the $c$-axis SAED diffraction spots also merits comment. Gaussian fits of the arc length of the second-order reflections have a range of full-width at halfmaximum (FWHM) of $7 \pm 2$ degrees (Figure S5). X-ray diffraction of samples recovered to ambient pressure probes longer length scales between $300 \mu \mathrm{m}$ (laboratory source) and $5 \mu \mathrm{m}$ (synchrotron); they show broadened arcs of similar widths: 8-12 degrees. ${ }^{5}$ However, in-situ xray diffraction measurements at high pressure, collected as nanothreads form from benzene, reveal much narrower arc widths of a degree or less. ${ }^{5}$ At these high pressures, volumetric packing efficiency is highly favored and may "squeeze out" gentle bends associated with on-thread structural defects. Consistent with this hypothesis, the rate of expansion of the inter-thread spacing greatly accelerates as the pressure is lowered below $3 \mathrm{GPa}^{5}$, perhaps reflecting the full expression of latent deformations that were squeezed out by enthalpic packing 
Stiff-Chiral-3 with Packing Disregistry

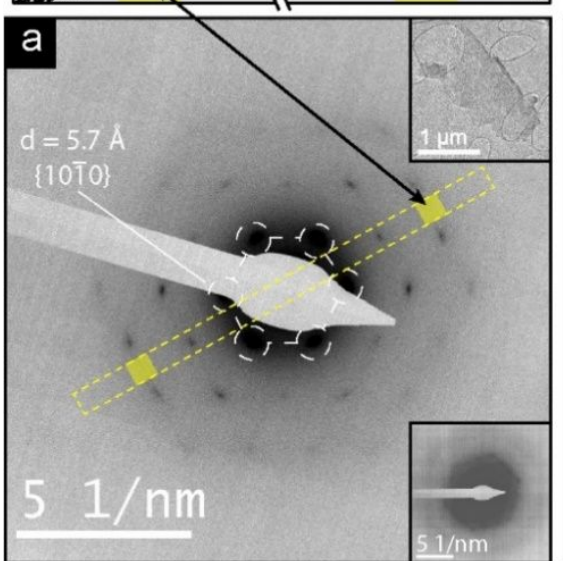

b

$\{1010\}$

$d=5.9 \AA$
Stiff-Chiral-3 with Packing Disregistry

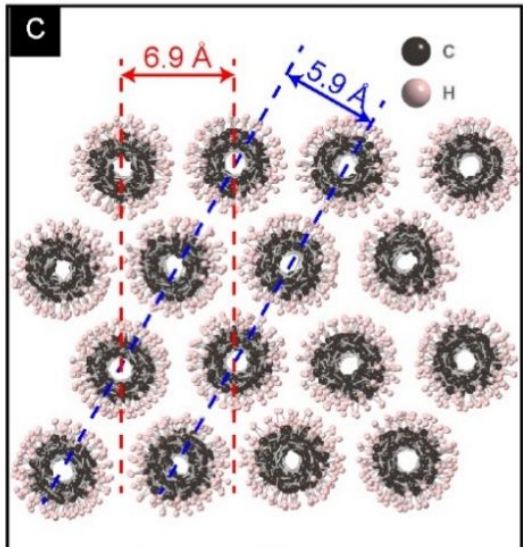

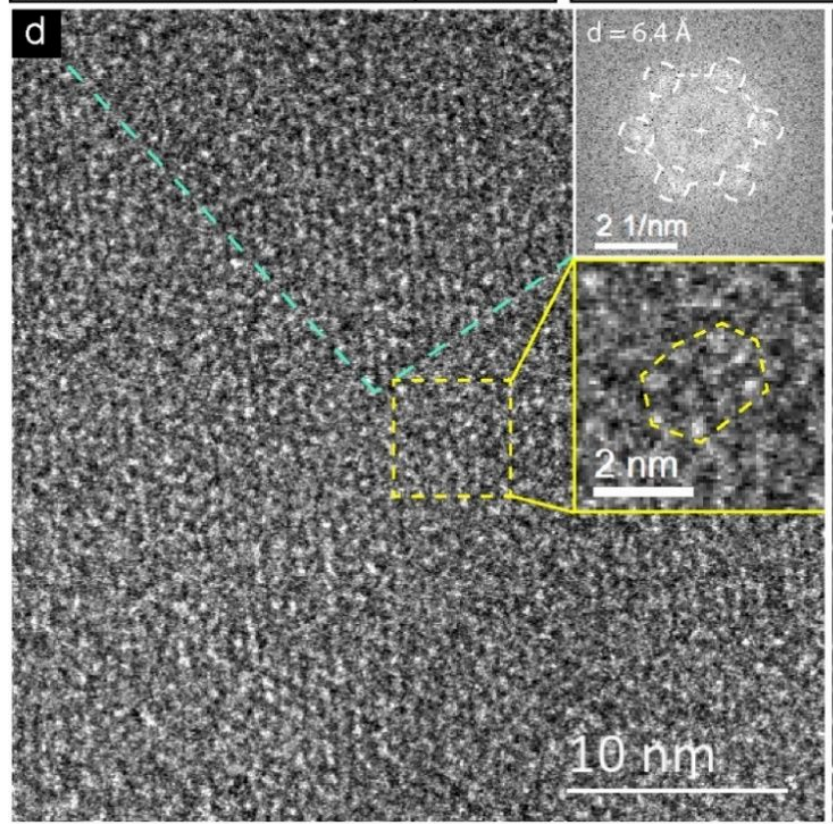

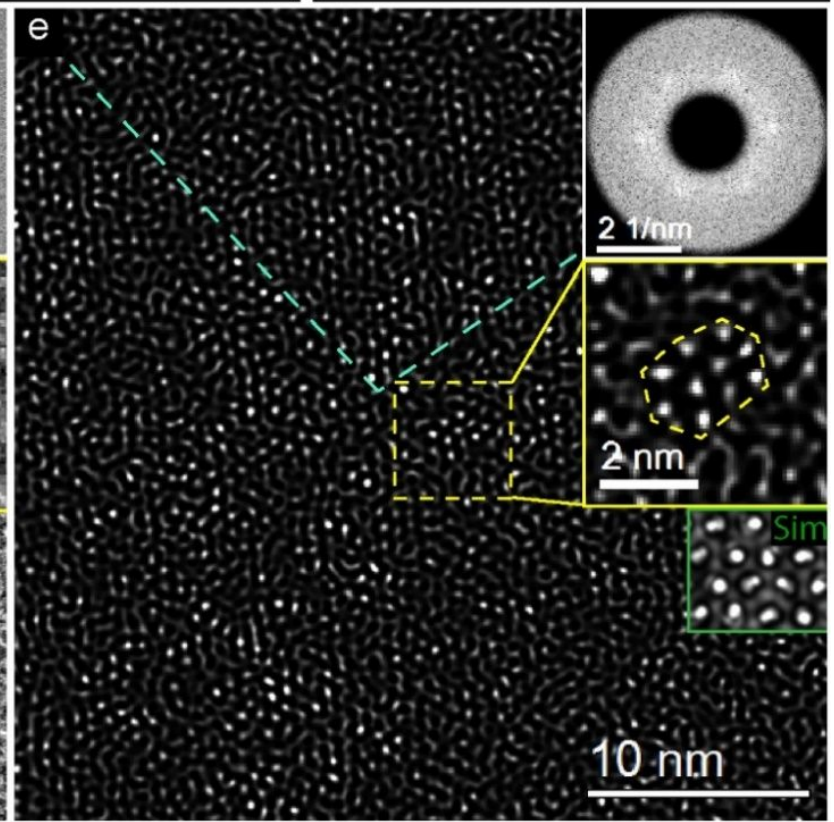

Figure 4. Nanothread Electron Diffraction Patterns and TEM Images. SAED pattern of nanothreads viewed along [0001] (a) is shown with an inset low-magnification TEM image of the particle on a lacey carbon TEM grid and an inset SAED pattern with increased contrast to emphasize a weak, diffuse "hexagonal halo" that appears near the location of the 10th-order reflections of the 2D pseudohexagonal packing diffraction pattern. The SAED patterns have inverted contrast to enhance the appearance of diffuse scattering that forms streaks between the spots, as shown in the line intensity profile above (a). The SAED pattern is shown in its original contrast and fully indexed in Figure S4. A simulated electron diffraction pattern and molecular model of hexagonally-packed stiff-chiral-3 with packing disregistry viewed along [0001] (b-c) are presented for comparison. Note that other instances of disregistry would yield streaks along additional symmetry directions. The (1010) diffraction plane (blue) and lattice parameter (red) are shown on the molecular model. An HREM image (d) and an IFT image (e) of nanothreads viewed along [0001] are shown with inset Fourier transforms. Magnified sections of each image highlight the appearance of well-aligned bundles of nanothreads. The region above the green dashed line shows striations in the vertical direction that may indicate 
bending of the threads away from the beam axis. An inset TEM image simulation of disordered class- 2 nanothreads with packing disregistry in the [0001] orientation (thickness $=96.61 \mathrm{~nm}$, defocus $=+8 \mathrm{~nm}$ ) is shown for comparison. The SAED pattern (a) and the HREM image (d) were taken from different particles of the same sample.

constraints at higher pressures. Alternatively, we note that residual benzene - frozen under compression - melts under decompression, ${ }^{5}$ and this phase change could produce inhomogeneous stresses on the lattice due to the associated volume or pressure changes.

In contrast to diffractive measurements, HREM imaging can reveal the local fine structure of the nanothread crystal at the level of individual threads. ${ }^{4-5}$ The pseudo-hexagonal crystalline packing of nanothreads in the [0001] $c$-axis zone is evident across areas on the order of a couple of hundred $\mathrm{nm}^{2}$ (Figure $4 \mathrm{~d}$ ). ${ }^{2} \quad$ A thread-level view of this packing is of much interest because nanothreads assemble into a high-symmetry pseudohexagonal lattice from an initial lower-symmetry monoclinic benzene molecular crystal while shrinking by as much as 40 to $50 \%$ in length as threads form. ${ }^{5}$ Individual nanothreads must extend parallel to [0001] through a substantial fraction of the $80-100 \mathrm{~nm}$ sample thickness (estimated by EELS) to produce the distinct points of contrast observed in the HREM image (Figure 4d). These columnar points are compelling evidence beyond diffraction that nanothreads have a one-dimensional structure with a large aspect ratio. The macroscopic spatial extent of the crystals of straight, relatively well-aligned nanothreads in the $a-b$ plane contrasts with the much smaller spatial extent of crystalline nanotube bundles. ${ }^{39}$ Large-scale twinning, which may add false symmetry to a diffraction pattern ${ }^{43}$ and confuse its interpretation, is not observed in the realspace imaging.

The reflections in the Fourier transform (FT) form a pseudo-hexagonal pattern with $6.4 \pm 0.2 \AA$ spacing (Figure $4 d$ inset), larger than measured by SAED (Figure 4a). Preliminary in-situ controlled-dose experiments reveal that this discrepancy is caused by lattice expansion due to accumulated electron dose over $30 \mathrm{e} / \AA^{2}{ }^{44}$ This expansion is irreversible and is a consequence of the damage introduced by the electron beam. The total electron dose of $150 \mathrm{e} / \AA^{2}$ used in Figure $4 \mathrm{~d}$ is significantly larger than the total dose used in the SAED (fluence below current measurement threshold). We further show below an HREM image with minimal beam damage ${ }^{33-35}$ (dose $=30$ $\mathrm{e} / \AA^{2}$ ) and a smaller interplanar spacing of $5.9 \AA$ for the thread bundle. Still, both the HREM image and the SAED pattern of Figure 4 are consistent with a well-crystallized pseudo-hexagonal packing.
To better reveal the details of thread packing, we performed Fourier-filtering on the image in Figure $4 d$ to obtain Figure 4e. The bright spots in the inverse Fourier transform (IFT) are arranged in a pseudo-hexagonal array with individual nanothreads deviating slightly from perfect alignment along the [0001] axis, which can be attributed to moderate bending. For example, in the top of Figure $4 \mathrm{e}$ a region of the crystal bends out of the c-axis enough to introduce subtle vertical linear striations. The anomalous nanothread lattice expansion observed during the last stage of decompression during unloading could be due to the release of latent bending (due to structural asymmetries or defects) suppressed under pressure. ${ }^{5}$ Moreover, the evaporation of benzene after decompression or the crushing of the nanothread samples before loading on the TEM grids could also be expected to bend them slightly. We simulated a TEM image from the relaxed supercell of disordered class- 2 nanothreads (Figure $4 \mathrm{e}$ inset). This model maintains full saturation while randomizing linkages between successive benzenederived subunits (see supporting information). The onthread disorder induces kinks and bends along the thread axis, ${ }^{4,26}$ which then disorder the projection of the nanothread lattice along the c-axis. We observe reasonable agreement between the simulated nanothreads with on-thread disorder and the experimental HREM images. Similar c-axis disorder could also be introduced by local variations in degree of saturation or domain boundaries between different thread types.

As Figure $4 \mathrm{~d}$ and Figure $4 \mathrm{e}$ indicate, the brightest points of the image tend to cluster together, suggesting that nanothreads form well-aligned nearly straight bundles of $\sim 10$ threads in small domains $\sim 2 \mathrm{~nm}$ in diameter (Figure $4 \mathrm{e}$ inset). A radially-averaged autocorrelation of the HREM image shows relatively strong autocorrelation out to second-nearest nanothreads $(\mathrm{d}=1.3 \mathrm{~nm})$, consistent with 'bundles' of this size (Figure S9). These small domains of near-ideal [0001] orientation are separated by similarlysized regions that are slightly out of alignment with the incident beam axis, and this structural scheme repeats across an area of several hundred square nanometers. In other words, a tight transversely short-ranged axial interthread alignment co-exists with a significant mosaic spread on longer length scales, with the entire structure maintaining a consistent overall axial alignment on the longest observable scales. The spots in the FT of the HREM 
2 image have an angular arc FWHM of $7 \pm 2$ degrees (Figure S10), in good agreement with the angular arc FWHM of the reflections in the SAED pattern (Figure S5). These observations suggest that the axis of the uniaxial pressure applied during synthesis ${ }^{5}$ is successful in imposing a consistent axial alignment on the longest transverse length scales. While packing constraints maintain tighter axial alignment on the smallest length scales, bending, twisting, tilting, and/or packing disclinations of nanothreads at local structural defects create modest deviations at intermediate length scales.

The structure of nanothreads along their axes was further examined by SAED from an orientation off the [0001] zone axis (Figure $5 \mathrm{a}$ and Figure $5 \mathrm{~b}$ ). Prior to this examination, the sample was annealed at $150^{\circ} \mathrm{C}$ in nitrogen atmosphere to improve its inter-thread registry in view of the weak van der Waals bonding between threads; modification of on-thread order associated with changes in covalent bonding seem less likely. It was difficult to find a zone axis that is perpendicular to [0001], such as $[10 \overline{10}]$ in the [0001] trace, ${ }^{5}$ in view of the limited time for SAED data collection before significant beam damage occurs. Thus, the angular orientation off [0001] could not be quantified. The SAED pattern in Figure 5a shows only one set of ( $\mathrm{h} 0 \overline{\mathrm{h}} \mathrm{0})$ Friedel pairs to the third order, consistent with a tilt away from [0001]. The $(10 \overline{1} 0)$ reflections imply a thread spacing of $5.8 \AA$, which agrees with the [1010] interplanar spacing of simulated pseudohexagonal nanothread packings (Figures S3-S4) for thread models with registry or disregistry. The 2 nd and 3 rd order reflections similarly match those in simulated diffraction patterns (Figures S11-S13).

Nanothreads that are in axial and/or azimuthal disregistry may also exhibit layer lines in their diffraction patterns (Figures S11- S13) that arise from the rotationally-averaged Fourier transform of
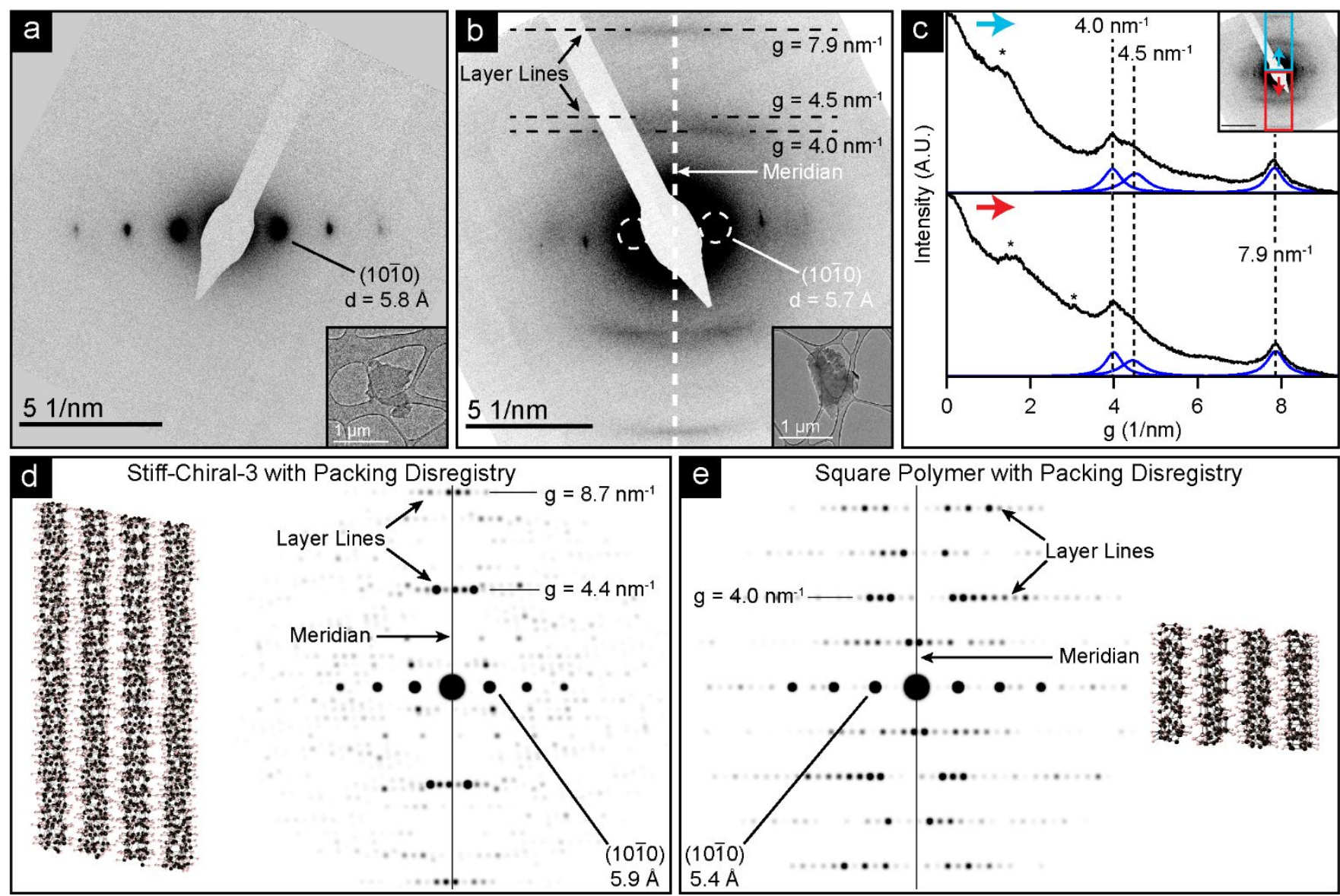

Figure 5. Nanothread Diffraction Patterns. (a) SAED pattern from nanothreads viewed from an orientation off the c-axis and with inset low-magnification TEM image of the particle on a lacey carbon grid. (b) SAED of annealed nanothreads viewed from an orientation off the $c$-axis with inset low magnification TEM image of the particle on a lacey carbon grid. The SAED patterns have 
inverted contrast to enhance the appearance of diffuse scattering, particularly in (b) where the diffuse scattering forms streaks on layer lines that are typical of fiber diffraction. (c) Linear intensity profiles drawn perpendicular to the layer lines reveal at least three layer lines at $\mathrm{g}=4.0,4.5$, and $7.9 \mathrm{~nm}^{-1}$ that indicate at least two distinct periodicities along the nanothread axes. The layer lines are fit with Lorentzian peaks shown in blue. Features that are associated with the beam blocker are marked with an asterisk $\left.{ }^{*}\right)$. The inset SAED pattern shows the positions and widths of the linear intensity profiles. A simulated electron diffraction pattern and model in orthographic projection of stiff-chiral-3 with packing disregistry in the $[01 \overline{1} 0]$ orientation (d) show layer lines that are similar to those observed in the experimental SAED pattern (see Figures S11-S13 for further examples). A simulated electron diffraction pattern and model in orthographic projection for square polymer are similarly shown (e). The stiff-chiral-3 diffraction pattern contains layer lines at $\mathrm{g}=4.4 \mathrm{~nm}^{-1}$ and $8.7 \mathrm{~nm}^{-1}$ with streaks that intersect the meridian, whereas the square polymer diffraction pattern reveals four layer lines, including a layer line at $\mathrm{g}=4.0 \mathrm{~nm}^{-1}$ with two streaks at $+/-25^{\circ}$ from the meridian. The additional layer lines contained in the diffraction pattern of square polymer may be too weak to observe in the experimental diffraction pattern.

individual nanothreads, much as the diffraction patterns of B-DNA (Figure S14) or carbon nanotubes reveal the Fourier transforms of the double helix or nanotube helices. 38,45 Layer lines were observed in the diffraction patterns of several of the annealed nanothread particles that were oriented off the [0001] axis, indicating a fiberlike diffraction (Figure $5 b$ ). The integrated linear intensity profiles in Figure $5 c$ allow extraction of layer line periodicities through peak fitting, which yields two peaks with $\mathrm{g}=4.0 \mathrm{~nm}^{-1}$ and $7.9 \mathrm{~nm}^{-1}$ and another peak with $\mathrm{g}$ $=4.5 \mathrm{~nm}^{-1}$. The FWHMs of these layer lines suggest coherence in the axial structural order across at least 13 to $18 \AA$ for this particle, a length spanned by approximately 6 to 9 linked benzenes (Figures S15-S16). We consider this calculation to be a lower bound to the intrinsic lengthscale of axial structural order, because additional factors (e.g. thermal diffuse scattering, electron beam damage) can also broaden the layer lines.

Two diffraction spots (elongated into streaks) are located on the layer line at $\mathrm{g}=4.0 \mathrm{~nm}^{-1}$ at $+/-25^{\circ}$ from the meridian - the vertical line through the origin that denotes the direction of the fiber axis (Figure 5b). Single streaks that intersect the meridian are observed on the layer lines at $\mathrm{g}=4.5 \mathrm{~nm}^{-1}$ and $7.9 \mathrm{~nm}^{-1}$. All the streaks on the layer lines at $\mathrm{g}=4.0$ and $4.5 \mathrm{~nm}^{-1}$ have interplanar spacings of 2.2-2.3 $\AA$ after accounting for azimuthal position, which is close to the

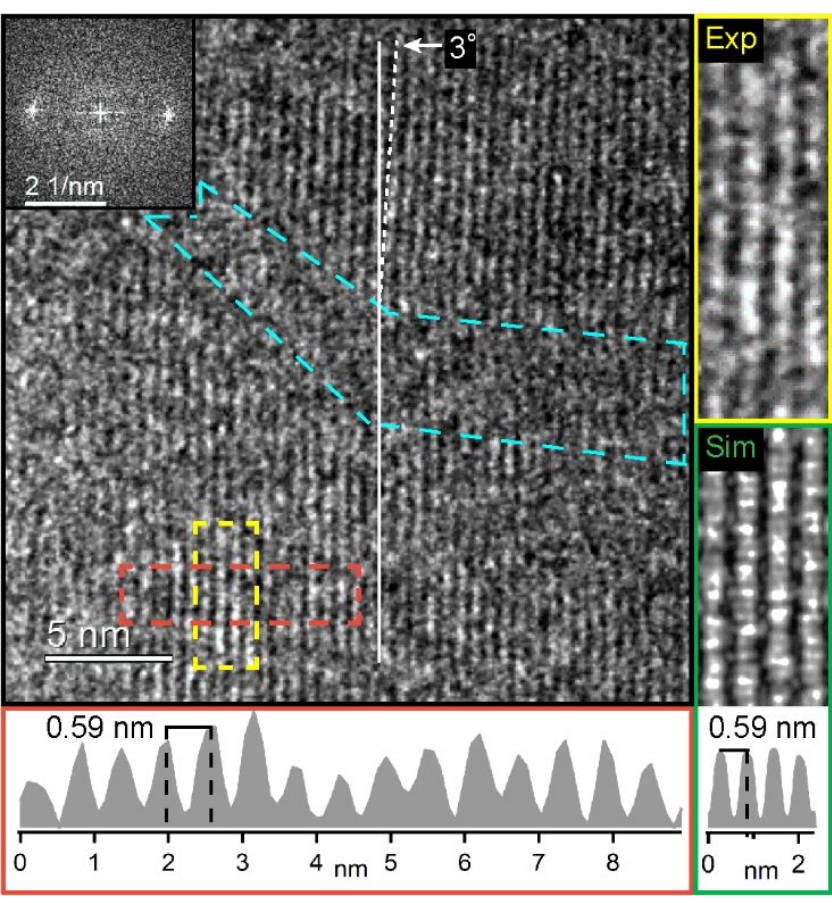

Figure 6. HREM image of nanothreads viewed from an orientation off the c-axis and with inset Fourier transform. A TEM image simulation of stiff-chiral-3 with packing disregistry (thickness $=79.97 \mathrm{~nm}$, defocus $=+40 \mathrm{~nm}$ ) is shown for comparison below a magnified region of the HREM image. Line profiles of the interthread spacing are shown below the HREM image and the image simulation. The dashed blue lines outline a region of low contrast in the nanothreads and mark the location of a $\sim 3^{\circ}$ bend in the nanothread bundle along the top line of the region.

distance between the neighboring linked benzene rings that compose the nanothreads. The disparate orientations of the streaks with 2.2-2.3 $\AA$ interplanar spacings indicate that the constituent benzene rings form distinct diffraction planes: one set of planes that are normal to the nanothread axis and two other sets of planes that are $+/-25^{\circ}$ from normal to the nanothread axis. Therefore, the 
2 two-fold and single streaks on the layer lines at $\mathrm{g}=4.0$ and $4.5 \mathrm{~nm}^{-1}$ likely originate from different nanothread structures that are present in the sample. For example, the simulated diffraction pattern for stiff-chiral-3 nanothreads with packing disregistry (Figure $5 d$ ) shows streaks that intersect the meridian on layer lines at $\mathrm{g}=4.4 \mathrm{~nm}^{-1}$ and $8.7 \mathrm{~nm}^{-1}$ but does not form two-fold streaks at $+/-25^{\circ}$ from the meridian. The streak on the layer line at $\mathrm{g}=4.4$ $\mathrm{nm}^{-1}$ is ubiquitous for chiral and disordered nanothread structures (Figure S11 and Figure S13), indicating that this interplanar spacing is the fundamental interplanar spacing of linked benzene units in nanothreads. On the other hand, simulated diffraction patterns show that achiral structures with packing disregistry, such as square polymer or polymer I (Figure S12), may form two streaks at $+/-25^{\circ}$ from the meridian along the layer line at $\mathrm{g}=4.0$ $\mathrm{nm}^{-1}$ (Figure 5e). The two-fold streaks are particularly interesting because they can only form when there is an achiral nanothread structure (Figure S12). The observation of layer lines provides the first evidence of structural order along the axes of nanothreads. We also note that stiffchiral-3 and square polymer are both suggested by an analysis of solid-state NMR data ${ }^{26}$ and that degree-4 threads (not analyzed here) could also be contributors to layer line features.

The absence of layer lines for other particles (Figure 5a) may be the result of a few factors, such as reduced onthread order (Figure S13), beam-induced sample damage, or orientation along a zone for which layer lines are not observed. The absence of distinct diffraction spots along the layer lines in the horizontal direction (Figure 5b) provides further evidence that the nanothread crystals have packing disregistry, as indicated by the simulations presented in Figures $\mathrm{S} 11-\mathrm{S} 13$ and exemplified by the simulated diffraction patterns of stiff-chiral-3 and square polymer nanothreads with packing disregistry shown in Figure $5 \mathrm{~d}$ and Figure $5 \mathrm{e}$. Diffraction simulations of nanothread models with packing registry show distinct reflections periodically spaced along the layer line direction, whereas nanothread models with packing disregistry show a multitude of weaker reflections along the layer lines that would smear into continuous streaks for larger (i.e. more realistic) simulated supercells.

HREM imaging of nanothreads in an orientation off the crystal $c$-axis - but again not necessarily perpendicular to [0001] - with minimal beam damage ${ }^{33-35}\left(\right.$ dose $\left.=30 \mathrm{e} / \AA^{2}\right)$ reveals an array of parallel threads (Figure 6). The interthread spacing is $5.9 \AA$, according to a line profile and the image FT (inset). This interthread spacing is smaller than both the $6.4-\AA$ spacing previously reported for imaging off $[0001]^{4}$ and the $6.4-\AA$ spacing for $c$-axis imaging shown in Figure $4 d$ at higher dose. The $5.9-\AA$ spacing in Figure 6 is similar to that seen in simulated TEM images of chiral nanothread structures with disregistry, such as stiff-chiral-3, (Figure 6 inset and Figure S18) and is closer to the interplanar spacing of $5.7 \AA$ observed for the first-order reflections in SAED patterns (Figure 4a, Figure $5 a$, and Figure $5 b$ ). The slight expansion in the lattice spacing from SAED to HREM imaging is likely due to beam-induced sample damage during imaging, which has been reported in organic materials. ${ }^{46-47} \mathrm{~A}$ more in-depth investigation of the effect of electron dose on the structural transformation of the nanothreads is required to further uncover the associated damage mechanism. ${ }^{44}$

We observe in the HREM image (Figure 6) two domains separated by a region of low contrast (outlined by green dashed lines) with a $\sim 3^{\circ}$ rotation between domains. This bending may contribute to the angular variations apparent from the arc length of the reflections in the [0001] SAED pattern (Figure 4a) and the bending in the thread columns apparent in the [0001] HREM image (Figure $4 d$ ). While the origin of this domain boundary is unclear, we note that the appearance of a region of lower image contrast is consistent with a twist or tilt of the nanothread packing axis at the transition between domains. ${ }^{48}$

We also observe "beading" associated with quasiperiodic variations of contrast along the axis of the nanothreads observed in HREM images (Figure 6). Just as for the images collected along [0001], such variations in contrast must be associated with a non-uniform local density of atoms in the sample. However, in contrast to the [0001] orientation, these image features are not associated with individual threads, but rather stacks of threads across the full thickness of the sample. To interpret the observed beading, we have performed TEM image simulations of a variety of nanothread models along the $[10 \overline{10}]$ zone across a range of thickness and defocus values (Figures S17-S26). For simulations of unit cells that consist of a single thread type with packing registry, we obtain consistent inter-bead distances of $0.5 \pm 0.1 \mathrm{~nm}$ across several different types of threads (Figure S27 and Figure S30). Similar simulations for unit cells that consist of a single thread type with packing disregistry show less regular inter-bead distances (Figure S28 and Figure S30). For comparison, experimental observations show irregular beading with inter-bead distances from 0.4 to $1.1 \mathrm{~nm}$ (Figure S29), which is similar to the less regular inter-bead distances of simulations with packing disregistry (Figure S30). On-thread disorder or a 
mixed population of different thread types can also contribute to variations in inter-bead spacing. The natural length-scale of the beading does provide some indication of the characteristic size of a structural subunit within the threads. The observed bead spacing of 4 to 11 angstroms thus corresponds to approximately 2 to 4 benzene subunits, which is comparable to the axial unit cells seen in the enumeration of candidate thread structures of degrees 4 and 6 (e.g. Figure S31).

\section{CONCLUSIONS}

This work has revealed the local structure of carbon nanothread crystals that is difficult to obtain from other characterization methods that probe the average structure over larger sample volumes. Nanothreads show a crystalline structure in their electron diffraction patterns with layer lines indicating order along their length. The hexagonal packing of individual thread columns parallel to the uniaxial axis of compression during synthesis is observed here in real space for the first time, in HREM imaging. We also observe subtle variations in this axial alignment of nanothreads, in which tight mutual correlations persist over 1-2 nm distances ("bundles") with some bounded angular wandering in the axial orientation over longer length scales. This axial alignment is likely formed during the synthesis of the nanothread crystal from polycrystalline, low-symmetry monoclinic solid benzene. The observation of [0001] columns provides strong evidence that nanothreads are indeed thread-like. Diffuse scattering observed in electron diffraction suggests disregistry in the pseudohexagonal packing, perhaps reminiscent of the merohedral disorder in alkalidoped fullerene crystals. ${ }^{49}$ Our study also shows that the interthread spacing observed in HREM images is consistent with an array of threads separated by van der Waals spacings.

HREM imaging off the [0001] zone furthermore reveals segments of the nanothread striations that exhibit quasiperiodic beading along their length. Low-dose HREM and electron diffraction provide structural information valuable to the goal of refining nanothread syntheses towards products of increasingly uniform structure and chemical hybridization. ${ }^{5}$ More studies need to be performed to explore the beam-induced damage mechanisms and the structural transformation of the nanothreads under the electron beam. In addition, more investigations are needed to explore the possibility of separating the crystals into individual threads for characterization of the atomic structure along their length.

\section{AUTHOR INFORMATION}

\section{Corresponding Author}

*nua10@psu.edu

\section{Present Address}

tDivision of Physics, Mathematics, and Astronomy, California Institute of Technology, Pasadena, CA, 91125, United States

\section{ACKNOWLEDGMENTS}

We acknowledge the Energy Frontier Research in Extreme Environments (EFree) Center, an Energy Frontier Research Center, funded by the Department of Energy Office (DOE) Office of Science (DE-SC0001057). We also acknowledge support from the Center for Nanothread Chemistry (CNC), funded by the National Science Foundation (CHE-1832471). Samples were synthesized at the Spallation Neutrons and Pressure beamline (SNAP) at the Spallation Neutron Source, a DOE Office of Science User Facility operated by Oak Ridge National Laboratory and the High-Pressure Neutron Diffractometer beamline (PLANET) at the Japan Proton Accelerator Research Complex. TEM was performed at the Materials Characterization Laboratory (MCL) in the Materials Research Institute (MRI) at the Pennsylvania State University (PSU). We acknowledge Dr. Ke Wang for his advice and assistance in performing TEM for this work.

\section{ASSOCIATED CONTENT}

\section{Supporting Information}

Supporting Information Available:

Experiment materials and methods, and theoretical modelling and simulation details, Figures S1-S34

This material is available free of charge via the internet at https://nam01.safelinks.protection.outlook.com/?url=http\%3 A\%2F\%2Fpubs.acs.org\&amp; data $=02 \% 7 C 01 \% 7$ Cnua $10 \% 40$ psu.edu\%7C29d28495c0af40102cff08d6ad69add2\%7C7cf48 d453ddb4389a9c1c115526eb52e\%7C0\%7C0\%7C636887066 731543997\&amp;sdata=VJbdh8qtqceBG\%2FkooBCvCJSLtW 2Cuy3xffI37thwCB0\%3D\&amp;reserved $=0$.

\section{References}

(1) Stojkovic, D.; Zhang, P.; Crespi, V. H. Smallest Nanotube: Breaking the Symmetry of $\mathrm{sp}^{3}$ Bonds in Tubular Geometries. Phys. Rev. Lett. 2001, 87, 125502.

(2) Wen, X. D.; Hoffmann, R.; Ashcroft, N. W. Benzene under High Pressure: A Story of Molecular Crystals Transforming to Saturated Networks, with a Possible Intermediate Metallic Phase. J. Am. Chem. Soc. 2011, 133, 9023-35.

(3) Olbrich, M.; Mayer, P.; Trauner, D. A Step Toward Polytwistane: Synthesis and Characterization of $\mathrm{C}_{2}$-Symmetric Tritwistane. Org. Biomol. Chem. 2014, 12, 108-12.

(4) Fitzgibbons, T. C.; Guthrie, M.; Xu, E. S.; Crespi, V. H.; 
Davidowski, S. K.; Cody, G. D.; Alem, N.; Badding, J. V. BenzeneDerived Carbon Nanothreads. Nat. Mater. 2015, 14, 43-7.

(5) Li, X.; Baldini, M.; Wang, T.; Chen, B.; Xu, E. S.; Vermilyea, B.; Crespi, V. H.; Hoffmann, R.; Molaison, J. J.; Tulk, C. A.; Guthrie, M.; Sinogeikin, S.; Badding, J. V. Mechanochemical Synthesis of Carbon Nanothread Single Crystals. J. Am. Chem. Soc. 2017, 139, 16343-16349.

(6) Xu, E. S.; Lammert, P. E.; Crespi, V. H. Systematic Enumeration of sp ${ }^{3}$ Nanothreads. Nano Lett. 2015, 15, 5124-30.

(7) Chen, B.; Hoffmann, R.; Ashcroft, N. W.; Badding, J.; Xu, E.; Crespi, V. Linearly Polymerized Benzene Arrays as Intermediates, Tracing Pathways to Carbon Nanothreads. J. Am. Chem. Soc. 2015, 137, 14373-86.

(8) Chen, B.; Wang, T.; Crespi, V. H.; Li, X.; Badding, J.; Hoffmann, R. All the Ways to Have Substituted Nanothreads. J. Chem. Theory Comput. 2018, 14, 1131-1140.

(9) Silveira, J. F.; Muniz, A. R. Functionalized Diamond Nanothreads from Benzene Derivatives. Phys. Chem. Chem. Phys. 2017, 19, 7132-7137.

(10) Li, X.; Wang, T.; Duan, P.; Baldini, M.; Huang, H. T.; Chen, B.; Juhl, S. J.; Koeplinger, D.; Crespi, V. H.; Schmidt-Rohr, K.; Hoffmann, R.; Alem, N.; Guthrie, M.; Zhang, X.; Badding, J. V. Carbon Nitride Nanothread Crystals Derived from Pyridine. J. Am. Chem. Soc. 2018, 140, 4969-4972.

(11) Nobrega, M. M.; Teixeira-Neto, E.; Cairns, A. B.; Temperini, M. L. A.; Bini, R. One-Dimensional Diamondoid Polyaniline-like Nanothreads from Compressed Crystal Aniline. Chem. Sci. 2018, 9, 254-260.

(12) Hong, G.; Diao, S.; Antaris, A. L.; Dai, H. Carbon Nanomaterials for Biological Imaging and Nanomedicinal Therapy. Chem. Rev. 2015, 115, 10816-906.

(13) Roman, R. E.; Kwan, K.; Cranford, S. W. Mechanical Properties and Defect Sensitivity of Diamond Nanothreads. Nano Lett. 2015, 15, 1585-90.

(14) Zhan, H.; Zhang, G.; Tan, V. B.; Cheng, Y.; Bell, J. M.; Zhang, Y. W.; Gu, Y. From Brittle to Ductile: A Structure Dependent Ductility of Diamond Nanothread. Nanoscale 2016, 8, 11177-84.

(15) Silveira, J. F. R. V.; Muniz, A. R. First-Principles Calculation of the Mechanical Properties of Diamond Nanothreads. Carbon 2017, 113, 260-265.

(16) Zhan, H. F.; Zhang, G.; Tan, V. B. C.; Cheng, Y.; Bell, J. M.; Zhang, Y. W.; Gu, Y. T. Diamond Nanothread as a New Reinforcement for Nanocomposites. Adv. Funct. Mater. 2016, 26, 5279-5283.

(17) Zhan, H.; Zhang, G.; Tan, V. B.; Gu, Y. The Best Features of Diamond Nanothread for Nanofibre Applications. Nat. Commun. 2017, 8, 14863.

(18) Duan, K.; Li, Y.; Li, L.; Hu, Y.; Wang, X. Diamond Nanothread Based Resonators: Ultrahigh Sensitivity and Low Dissipation. Nanoscale 2018, 10, 8058-8065.

(19) Wu, W. K.; Tai, B.; Guan, S.; Yang, S. Y. A.; Zhang, G. Hybrid Structures and Strain-Tunable Electronic Properties of Carbon Nanothreads. J. Phys. Chem. C 2018, 122, 3101-3106.

(20) Gao, J.; Zhang, G.; Yakobson, B. I.; Zhang, Y. W. Kinetic Theory for the Formation of Diamond Nanothreads with Desired
Configurations: A Strain-Temperature Controlled Phase Diagram. Nanoscale 2018, 10, 9664-9672.

(21) Zhan, H. F.; Zhang, G.; Zhang, Y. Y.; Tan, V. B. C.; Bell, J. M.; Gu, Y. T. Thermal Conductivity of a New Carbon Nanotube Analog: The Diamond Nanothread. Carbon 2016, 98, 232-237.

(22) Zhu, T.; Ertekin, E. Phonons, Localization, and Thermal Conductivity of Diamond Nanothreads and Amorphous Graphene. Nano Lett. 2016, 16, 4763-72.

(23) Wheeldon, I.; Minteer, S. D.; Banta, S.; Barton, S. C.; Atanassov, P.; Sigman, M. Substrate Channelling as an Approach to Cascade Reactions. Nat. Chem. 2016, 8, 299-309.

(24) Habibi, Y.; Lucia, L. A.; Rojas, O. J. Cellulose Nanocrystals: Chemistry, Self-Assembly, and Applications. Chem. Rev. 2010, 110, 3479-500.

(25) Li, H. Y.; Carter, J. D.; LaBean, T. H. Nanofabrication by DNA Self-Assembly. Mater. Today 2009, 12, 24-32.

(26) Duan, P.; Li, X.; Wang, T.; Chen, B.; Juhl, S. J.; Koeplinger, D.; Crespi, V. H.; Badding, J. V.; Schmidt-Rohr, K. The Chemical Structure of Carbon Nanothreads Analyzed by Advanced SolidState NMR. J. Am. Chem. Soc. 2018, 140, 7658-7666.

(27) Wang, T.; Duan, P.; Xu, E. S.; Vermilyea, B.; Chen, B.; Li, X.; Badding, J. V.; Schmidt-Rohr, K.; Crespi, V. H. Constraining Carbon Nanothread Structures by Experimental and Calculated Nuclear Magnetic Resonance Spectra. Nano Lett. 2018, 18, 4934-4942.

(28) Iijima, S.; Ichihashi, T. Single-Shell Carbon Nanotubes of 1-nm Diameter. Nature 1993, 363, 603-605.

(29) Harris, J. P. Transmission Electron Microscopy of Carbon: A Brief History. C 2018, $4,4$.

(30) Boudet, A.; Kubin, L. P. The Limitations to Resolution in the Observation of Radiation-Sensitive Specimens by Electron Microscopy. Ultramicroscopy 1982, 8, 409-416.

(31) Egerton, R. F.; Li, P.; Malac, M. Radiation Damage in the TEM and SEM. Micron 2004, 35, 399-409.

(32) Libera, M. R.; Egerton, R. F. Advances in the Transmission Electron Microscopy of Polymers. Polym. Rev. 2010, 50, 321-339.

(33) Barton, B.; Jiang, B.; Song, C.; Specht, P.; Calderon, H.; Kisielowski, C. Atomic Resolution Phase Contrast Imaging and Inline Holography Using Variable Voltage and Dose Rate. Microsc. Microanal. 2012, 18, 982-94.

(34) Lolla, D.; Gorse, J.; Kisielowski, C.; Miao, J.; Taylor, P. L.; Chase, G. G.; Reneker, D. H. Polyvinylidene Fluoride Molecules in Nanofibers, Imaged at Atomic Scale by Aberration Corrected Electron Microscopy. Nanoscale 2016, 8, 120-8.

(35) Kisielowski, C.; Specht, P.; Gygax, S. M.; Barton, B.; Calderon, H. A.; Kang, J. H.; Cieslinski, R. Instrumental Requirements for the Detection of Electron Beam-Induced Object Excitations at the Single Atom Level in High-Resolution Transmission Electron Microscopy. Micron 2015, 68, 186-193.

(36) Brydson, R.; Zhili, Z.; Brown, A. In Revisiting the Determination of Carbon $s p^{2} / s p^{3}$ Ratios via Analysis of the EELS Carbon K-edge, EMC 2008, Berlin, Heidelberg, Luysberg, M.; Tillmann, K.; Weirich, T., Eds. Springer Berlin Heidelberg: Berlin, Heidelberg, 2008; pp 357-358.

(37) Urbonaite, S.; Wachtmeister, S.; Mirguet, C.; Coronel, E.; Zou, W. Y.; Csillag, S.; Svensson, G. EELS Studies of Carbide Derived Carbons. Carbon 2007, 45, 2047-2053. 
(38) Chandrasekaran, R.; Stubbs, G. Fibre Diffraction. In Int. Tables Crystallogr., Arnold, E.; Himmel, D. M.; Rossmann, M. G., Eds. Chester, England, 2012; Vol. F.

(39) Thess, A.; Lee, R.; Nikolaev, P.; Dai, H.; Petit, P.; Robert, J.; Xu, C.; Lee, Y. H.; Kim, S. G.; Rinzler, A. G.; Colbert, D. T.; Scuseria, G. E.; Tomanek, D.; Fischer, J. E.; Smalley, R. E. Crystalline Ropes of Metallic Carbon Nanotubes. Science 1996, 273, 483-7.

(40) Cornell, W. D.; Cieplak, P.; Bayly, C. I.; Gould, I. R.; Merz, K. M.; Ferguson, D. M.; Spellmeyer, D. C.; Fox, T.; Caldwell, J. W.; Kollman, P. A. A Second Generation Force Field for the Simulation of Proteins, Nucleic Acids, and Organic Molecules. J. Am. Chem. Soc. 1996, 118, 2309-2309.

(41) Plimpton, S. Fast Parallel Algorithms for Short-Range Molecular Dynamics. J. Comput. Phys. 1995, 117, 1-19.

(42) Launois, P.; Ravy, S.; Moret, R. Diffuse Scattering and Orientational Correlations in Solid $C_{60}$. Phys. Rev. B 1995, 52, 5414-5425

(43) Kiho, H.; Peterlin, A.; Geil, P. H. Polymer Deformation. VI. Twinning and Phase Transformation of Polyethylene Single
Crystals as a Function of Stretching Direction. J. Appl. Phys. 1964, 35, 1599-1605.

(44) Juhl, S.; Li, X.; Badding, J.; Alem, N. Low Dose Characterization of Diamondoid Carbon Nanothreads by Transmission Electron Microscopy. Microsc. Microanal. 2017, 23 , 1846-1847.

(45) Lucas, A. A.; Lambin, P. Diffraction by DNA, Carbon Nanotubes and Other Helical Nanostructures. Rep. Prog. Phys. 2005, 68, 1181-1249.

(46) Kumar, S.; Adams, W. W. Electron Beam Damage in High Temperature Polymers. Polymer 1990, 31, 15-19.

(47) Grubb, D. T. Radiation Damage and Electron Microscopy of Organic Polymers. J. Mater. Sci. 1974, 9, 1715-1736.

(48) Martin, D. C.; Thomas, E. L. Grain Boundaries in ExtendedChain Polymers: Theory and Experiment. Philos. Mag. A 1991, 64, 903-922.

(49) Stephens, P. W.; Mihaly, L.; Lee, P. L.; Whetten, R. L.; Huang, S. M.; Kaner, R.; Deiderich, F.; Holczer, K. Structure of Single-Phase Superconducting K3c60. Nature 1991, 351, 632-634. 


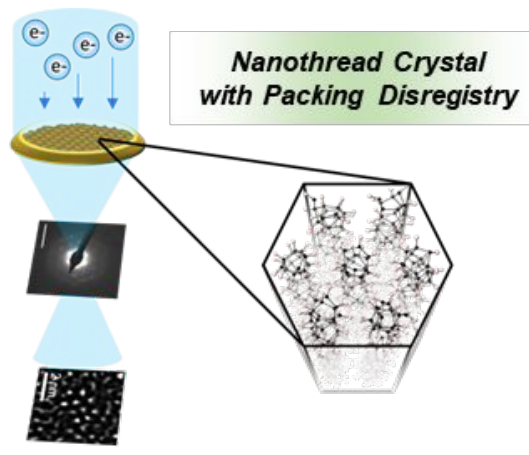

For Table of Contents Only 